\title{
Mathematical Modeling of the Acoustic Phonons Spectra Arising in Multilayer Nanostructures
}

\author{
Igor Boyko \\ Department of Software Engineering \\ Ternopil Ivan Puluj National Technical \\ University \\ Ternopil, Ukraine \\ boyko.i.v.theory@gmail.com \\ Yuriy Stoianov \\ Department of Software Engineering \\ Ternopil Ivan Puluj National Technical University \\ Ternopil, Ukraine \\ yuriy556s@gmail.com
}

\author{
Iaroslav Kinakh \\ Department of Software Engineering \\ Ternopil Ivan Puluj National Technical \\ University \\ Ternopil, Ukraine \\ kinakh@live.com
}

\author{
Taras Byts \\ Department of Software Engineering \\ Ternopil Ivan Puluj National Technical University \\ Ternopil, Ukraine \\ byts.taras@gmail.com
}

\begin{abstract}
Based on a modification of the elastic continuum model, a mathematical model describing the processes of the emergence and propagation of acoustic phonons in multilayer planar semiconductor nanostructures was constructed. Using the obtained mathematical model, supplemented by the boundary conditions for medium displacements and components of the elastic tensor at the boundaries of the studied nanostructure, the theory of the spectrum was developed and the phonon modes were normalized. Using the geometric and physical parameters of a typical nanostructure, we calculated and simulated the spectral characteristics of acoustic phonons. The obtained results can be used for further research and mathematical modeling of the electron-phonon interaction subsidence in complex semiconductor nanoheterosystems, which are the basic elements of quantum cascade lasers and detectors.
\end{abstract}

Keywords - elastic continuum model, acoustic phonon, multilayer nanostructure, normalization condition, dispersion equation, finite difference method.

\section{INTRODUCTION}

The rapid development of modern semiconductor nanotechnology [1-3] determines their active use in various fields of human technological activity, science and engineering.

In particular, in information technologies, semiconductor nanoscale objects are the precision and functional parts of triggers, logical elements [4], which is the basic basis for the development of quantum computers and quantum computing $[5,6]$.

Quantum-cascade lasers (QCL) [7, 8] created on the basis of plane semiconductor resonant-tunnel nanosystems (RTS), having the ability to work in different ranges of electromagnetic waves, are widely used: from medicine to energy technologies in solar batteries. On the other hand, quantum cascade detectors (QCD) of the near and middle infrared range of electromagnetic waves have the ability to work in the so-called "transparency windows" of the earth's atmosphere, so they are directly used in military technologies for locating objects (anti-Stealth technology).

The functional elements of the nanodevices mentioned above, in particular, QCL and QCD are semiconductor RTS arsenide ( GaAs, AlAs, GaAlAs ) [7] or nitride type ( GaN, AlN, GaAlN ). [8]. Therefore, theoretical research and mathematical modeling of physical processes (in particular, those related to dissipation of electron fluxes on phonons, impurities, dislocations) occurring in such nanoRTS is necessary to ensure the conditions for efficient operation of the above-mentioned nanodevices.

One of these urgent tasks still unresolved is the study of the processes of the emergence and propagation of acoustic phonons in multilayer nanostructures. It should be noted that for this direction, adjacent model calculations were performed only for nanostructures with single potential wells [9].

In this case, a characteristic feature of the models mentioned is that the components of the stress tensor on the walls of quantum wells were considered equal to zero, that is: $\sigma_{i j}=0, i, j=1,2,3$. Since, as was established in [10], for an effective description and simulation of physical processes occurring in QCL and QCD, it is necessary to consider a separate cascade of these nano-devices in the model of an open nanostructure, then the models proposed in the mentioned above works cannot be used.

Thus, it should be concluded that the models constructed and investigated in [9] are a very rough approximation, and the theory and results obtained in them cannot be applied to the study of experimentally created multilayer nanostructures - active elements of QCL and QCD cascades. The modified mathematical model of acoustic phonons for plane multilayered nanosystems should take into account the fact that the components $\sigma_{i j}$ of the stress tensor should monotonously decrease in the medium to the left and right of the studied nanosystem.

In the proposed paper, a mathematical model which describes the behavior and spectral characteristics of acoustic phonons arising in the open plane multilayer semiconductor nanostructure is constructed. Using the elastic continuum model and the boundary conditions for the constituent acoustic phonon modes and the stress tensor components, analytical and numerical solutions of differential equations describing the elastic displacement of the layers medium of the studied RTS are obtained. For the obtained types of acoustic phonon modes, their normalization was performed.

The constructed mathematical model and the developed theory of acoustic modes can be applied to further study phonon and electronic processes in multilayer semiconductor nanostructures. 


\section{Statement of The Problem. Components of the DISPLACEMENT FIELD FOR THE MEDIA OF THE NANOSYSTEM LAYERS}

To build a mathematical model of the proposed problem, we consider on the conditions and processes leading to the appearance of acoustic phonon modes arising inside the multilayer semiconductor nanosystem of $\mathrm{AlN} / \mathrm{GaN}$ nitride alloy. Taking into account the designations made in Fig.1, the studied nanosystem is placed in such a way that the coordinate axis $x_{3}$ is perpendicular to the heteroboundaries of the layers of the nanosystem media.

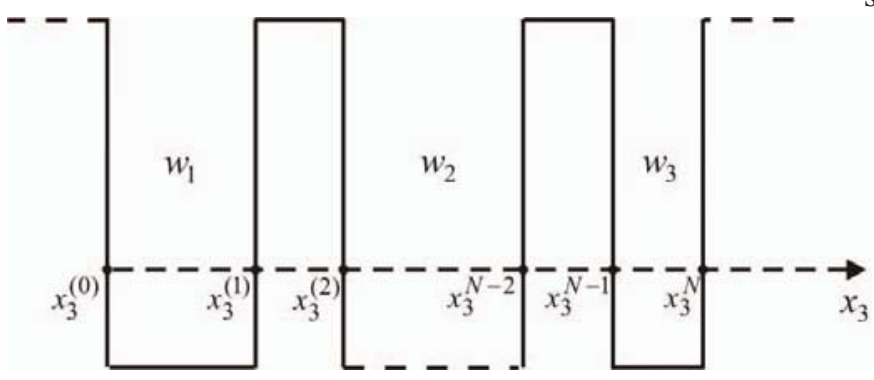

Fig. 1. Geometric scheme of plane multilayered semicondutor nanostructure

Thus, the equation of motion for the elastic displacement within the nanosystem is written in next form:

$$
\begin{gathered}
\rho\left(x_{3}\right) \frac{\partial^{2} u_{i}\left(x_{1}, x_{2}, x_{3}, t\right)}{\partial t^{2}}=\frac{\partial \sigma_{i k}\left(x_{1}, x_{2}, x_{3}\right)}{\partial x_{k}} \\
i, k=(1 ; 2 ; 3)
\end{gathered}
$$

where it is taken into account that:

$$
\begin{aligned}
& \rho\left(x_{3}\right)=\sum_{p=1}^{N+1} \rho^{(p)}\left[\theta\left(x_{3}-x_{3}^{(p-1)}\right)-\theta\left(x_{3}-x_{3}^{(p)}\right)\right] \\
& x_{3}^{(-1)}=-\infty ; x_{3}^{(N+1)}=+\infty .
\end{aligned}
$$

$x_{3}$ - coordinate dependent density of the nanostructure medium, $u_{i}=u_{i}\left(x_{1}, x_{2}, x_{3}, t\right)$ - component of the elastic displacement vector of the medium at the point $r=\left(x_{1}, x_{2}, x_{3}\right)$ at the moment of time $t$,

$$
\sigma_{i k}\left(r, x_{3}\right)=C_{i k l m}\left(x_{3}\right) u_{l m}(r), l, m=(1 ; 2 ; 3),
$$

- stress tensor,

$$
C_{i k l m}\left(x_{3}\right)=\sum_{p=1}^{N+1} C_{i k l m}^{(p)}\left[\theta\left(x_{3}-x_{3}^{(p-1)}\right)-\theta\left(x_{3}-x_{3}^{(p)}\right)\right],
$$

where as a result of different kinds of the nanosystem material layers the elastic constants $C_{i k l m}$ is depending on the coordinate $x_{3}$,

$$
u_{l m}(r)=\frac{1}{2}\left(\frac{\partial u_{l}(r)}{\partial x_{m}}+\frac{\partial u_{m}(r)}{\partial x_{l}}\right)
$$

- components of the strain tensor.

Given the formulation of the problem, we will assume that the formation of the acoustic waves propagation occurs in the direction of the axis $O x_{1}$. In this case, the cross section of an arbitrary $p$ - th layer of the nanostructure is homogeneous. This allows, separating the variables, to search for solutions of equation (1) in the form :

$$
\begin{gathered}
u_{i}(r, t)=u_{i}\left(x_{1}, x_{3}, t\right)=\sum_{p=1}^{N}\left[\begin{array}{l}
u_{1}^{(p)}\left(x_{3}\right) \\
u_{2}^{(p)}\left(x_{3}\right) \\
u_{3}^{(p)}\left(x_{3}\right)
\end{array}\right] \times \\
\times\left[\theta\left(x_{3}-x_{3}^{(p-1)}\right)-\theta\left(x_{3}-x_{3}^{(p)}\right)\right] e^{i\left(\omega t-q x_{1}\right)},
\end{gathered}
$$

where $u_{1}^{(p)}\left(x_{3}\right), u_{2}^{(p)}\left(x_{3}\right), u_{3}^{(p)}\left(x_{3}\right)$ is the components of the displacement vector within the selected $p$-th layer of the nanosystem. Now the equation of motion (1), taking into account relations (3) - (6), takes the form:

$$
\left[\rho^{(p)} \omega^{2} \delta_{k i}-C_{i k l m}^{(p)} \frac{\partial^{2}}{\partial x_{k} \partial x_{l}}\right]\left[\begin{array}{l}
u_{1}^{(p)}\left(x_{3}\right) \\
u_{2}^{(p)}\left(x_{3}\right) \\
u_{3}^{(p)}\left(x_{3}\right)
\end{array}\right] e^{i\left(\omega t-q x_{1}\right)}=0
$$

Taking into account the kind of elastic elasticity tensor $C_{i k l m}^{(p)}$ for a wurtzite-type crystal lattice, turning to tensor Voigt notation, from (7), we obtain three differential equations describing the acoustic modes arising in an arbitrary layer of nanostructure:

$$
\begin{gathered}
\frac{d^{2} u_{1}^{(p)}\left(x_{3}\right)}{d x_{3}^{2}}-i q\left(1+\frac{C_{13}^{(p)}}{C_{44}^{(p)}}\right) \frac{d u_{3}^{(p)}\left(x_{3}\right)}{d x_{3}}+ \\
+\left(\frac{\rho^{(p)} \omega^{2}-q^{2} C_{11}^{(p)}}{C_{44}^{(p)}}\right) u_{1}^{(p)}\left(x_{3}\right)=0,
\end{gathered}
$$

$$
\frac{d^{2} u_{2}^{(p)}\left(x_{3}\right)}{d x_{3}^{2}}-\left(\frac{q^{2} C_{66}^{(p)}-\rho^{(p)} \omega^{2}}{C_{44}^{(p)}}\right) u_{2}^{(p)}\left(x_{3}\right)=0,
$$

$$
\begin{gathered}
\frac{d^{2} u_{3}^{(p)}\left(x_{3}\right)}{d x_{3}^{2}}-i q\left(\frac{C_{13}^{(p)}+C_{44}^{(p)}}{C_{33}^{(p)}}\right) \frac{d u_{1}^{(p)}\left(x_{3}\right)}{d x_{3}}+ \\
+\left(\frac{\rho^{(p)} \omega^{2}-q^{2} C_{44}^{(p)}}{C_{33}^{(p)}}\right) u_{3}^{(p)}\left(x_{3}\right)=0
\end{gathered}
$$


III. BOUNDARY CONDITIONS AND THE SOLUTIONS OF EQUATIONS DESCRIBING THE MODEL OF ACOUSTIC PHONONS IN A MULTILAYER NANOSTRUCTURE
From the continuity conditions for the elastic tensor components $\sigma_{x_{1} x_{3}}, \sigma_{x_{3} x_{3}},, \sigma_{x_{2} x_{3}}$ and the components of the elastic displacement, the boundary conditions are obtained.

$$
\left[\begin{array}{l}
\left.u_{\alpha}^{(p)}\left(q, \omega, x_{3}\right)\right|_{x_{3}=x_{3}^{(p)}-\varepsilon}=\left.u_{\alpha}^{(p+1)}\left(q, \omega, x_{3}\right)\right|_{x_{3}=x_{3}^{(p)}+\varepsilon} ; \alpha=1,2,3 ; \varepsilon \rightarrow 0 \\
\left.C_{44}^{(p)} \frac{d u_{2}^{(p)}\left(x_{3}\right)}{d x_{3}}\right|_{x_{3}=x_{3}^{(p)}-\varepsilon}=\left.C_{44}^{(p+1)} \frac{d u_{2}^{(p+1)}\left(x_{3}\right)}{d x_{3}}\right|_{x_{3}=x_{3}^{(p)}+\varepsilon} ; \\
\left.C_{44}^{(p)}\left(i q u_{3}^{(p)}\left(x_{3}\right)+\frac{d u_{1}^{(p)}\left(x_{3}\right)}{d x_{3}}\right)\right|_{x_{3}=x_{3}^{(p)}-\varepsilon}=\left.C_{44}^{(p+1)}\left(i q u_{3}^{(p+1)}\left(x_{3}\right)+\frac{d u_{1}^{(p+1)}\left(x_{3}\right)}{d x_{3}}\right)\right|_{x_{3}=x_{3}^{(p)}+\varepsilon} \\
\left(\begin{array}{c}
\left.-i q C_{13}^{(p)} u_{1}^{(p)}\left(x_{3}\right)+C_{33}^{(p)} \frac{d u_{3}^{(p)}\left(x_{3}\right)}{d x_{3}}\right)\left.\right|_{x_{3}=x_{3}^{(p)}-\varepsilon}=\left.\left(-i q C_{13}^{(p+1)} u_{1}^{(p+1)}\left(x_{3}\right)+C_{33}^{(p+1)} \frac{d u_{3}^{(p)}\left(x_{3}\right)}{d x_{3}}\right)\right|_{x_{3}=x_{3}^{(p)}+\varepsilon}
\end{array} .\right.
\end{array}\right.
$$

In addition, due to the properties of the studied nanosystem model, the conditions for the zero component of the elastic displacement, that is:

$$
\left.u_{\alpha}\left(q, \omega, x_{3}\right)\right|_{x_{3} \rightarrow \pm \infty} \rightarrow 0 ; \alpha=1,2,3
$$

Solutions of equation (9) can be obtained analytically, taking into account the condition (12) is as follows:

$$
\begin{gathered}
u_{2}\left(q, \omega, x_{3}\right)=u_{2}^{(0)}\left(q, \omega, x_{3}\right) \theta\left(-x_{3}\right)+u_{2}^{(N+1)}\left(q, \omega, x_{3}\right) \theta\left(x_{3}-x_{3}^{N}\right)+\sum_{p=1}^{N} u_{2}^{(p)}\left(q, \omega, x_{3}\right)\left[\theta\left(x_{3}-x_{3}^{p-1}\right)-\theta\left(x_{3}-x_{3}^{p}\right)\right]= \\
=B_{2}^{(0)} e^{i k_{2}^{(0)}(q, \omega) x_{3}} \theta\left(-x_{3}\right)+\sum_{p=1}^{N}\left[A_{2}^{(p)} e^{-i \chi_{2}^{(p)}(q, \omega) x_{3}}+B_{2}^{(p)} e^{\left.i \chi_{2}^{(p)}(q, \omega) x_{3}\right]}\right]\left[\theta\left(x_{3}-x_{3}^{p-1}\right)-\theta\left(x_{3}-x_{3}^{p}\right)\right]+ \\
+A_{2}^{(N+1)} e^{-i k_{2}^{(N+1)}(q, \omega)\left(x_{3}-x_{3}^{N}\right)} \theta\left(x_{3}-x_{3}^{N}\right), k_{2}^{(p)}(q, \omega)=\sqrt{\frac{\rho^{(p)} \omega^{2}-q^{2} C_{66}^{(p)}}{C_{44}^{(p)}}},
\end{gathered}
$$

where the coefficients $B_{2}^{(0)}, A_{2}^{(p)}, B_{2}^{(p)}, A_{2}^{(N+1)}$ are determined with the first and second conditions (11).

Solutions of the system of equations (8) and (10) with regard to the first, third and fourth conditions (11) and conditions (12) are found using the finite difference method, using approximation of derivatives as:

$$
\begin{aligned}
& \left.\frac{d u_{\alpha}\left(q, \omega, x_{3}\right)}{d x_{3}}\right|_{x_{3}=x_{3}^{(s)}}=\frac{u_{\alpha}^{(s+1)}-u_{\alpha}^{(s)}}{h} ; \\
& \left.\frac{d^{2} u_{\alpha}\left(q, \omega, x_{3}\right)}{d z^{2}}\right|_{x_{3}=x_{3}^{(s)}}=\frac{u_{\alpha}^{(s+1)}-2 u_{\alpha}^{(s)}+u_{\alpha}^{(s-1)}}{h^{2}}
\end{aligned}
$$

Then on a uniform grid:

$$
\bar{\Omega}=\left\{x_{3}^{(s)}=s h, s=0,1, \ldots N, h=\frac{l}{N}\right\}
$$

where $l=x_{3}^{N}$ - is the total thickness of the RTS, the difference scheme is obtained:

$$
\left[\begin{array}{l}
u_{1}^{(s+1)}-\left(2+h^{2} b_{1}(q, \omega)\right) u_{1}^{(s)}+u_{1}^{(s-1)}-h a_{1} u_{3}^{(s+1)}+h a_{1} u_{3}^{(s)}=0 \\
u_{3}^{(s+1)}-\left(2+h^{2} b_{2}(q, \omega)\right) u_{3}^{(s)}+u_{3}^{(s-1)}-h a_{2} u_{1}^{(s+1)}-h a_{2} u_{1}^{(s)}=0 \\
c_{1} u_{1}^{(s+1)}+\left(1-c_{1}\right) u_{1}^{(s)}-u_{1}^{(s-1)}+i q h c_{1} u_{3}^{(s+1)}-i q h u_{3}^{(s)}=0 ; \\
i q h c_{3} u_{1}^{(s+1)}+i q h u_{1}^{(s)}-c_{4} u_{3}^{(s+1)}+\left(c_{2}+c_{4}\right) u_{3}^{(s)}-c_{2} u_{3}^{(s-1)}=0 ; \\
u_{1}^{(0)}=u_{1}^{(N+1)}=0 ; u_{3}^{(0)}=u_{3}^{(N+1)}=0
\end{array}\right.
$$

from which the phonon spectrum $\omega_{n}$ and phonon modes $u_{1}\left(q, \omega, x_{3}\right), u_{3}\left(q, \omega, x_{3}\right)$ are obtained. In relation (15) the following notation is made: 


$$
\begin{gathered}
c_{1}=\frac{C_{44}^{(p+1)}}{C_{44}^{(p)}} ; c_{2}=\frac{C_{33}^{(p)}}{C_{13}^{(p)}} ; c_{3}=\frac{C_{13}^{(p+1)}}{C_{13}^{(p)}} ; c_{4}=\frac{C_{33}^{(p+1)}}{C_{13}^{(p)}} ; \\
a_{1}=i q\left(1+\frac{C_{13}^{(p)}}{C_{44}^{(p)}}\right) ; b_{1}(q, \omega)=\frac{q^{2} C_{11}^{(p)}-\rho^{(p)} \omega^{2}}{C_{44}^{(p)}} ; \\
a_{2}=i q \frac{\left(C_{13}^{(p)}+C_{44}^{(p)}\right)}{C_{33}^{(p)}} .
\end{gathered}
$$

Phonon mode normalization is performed according to the ratios:

$$
\int_{0}^{x_{3}^{N}} \rho\left(x_{3}\right)\left|u_{2}\left(q, \omega, x_{3}\right)\right|^{2} d x_{3}=\frac{\hbar}{2 \omega_{n} S}
$$

and

$$
\int_{0}^{x_{3}^{N}} \rho\left(x_{3}\right)\left(\left|u_{1}\left(q, \omega, x_{3}\right)\right|^{2}+\left|u_{3}\left(q, \omega, x_{3}\right)\right|^{2}\right) d x_{3}=\frac{\hbar}{2 \omega_{n} S},
$$

$S$ - the nanostructure cross-sectional area.

\section{DISCUSSION OF THE RESULTS}

Using the theory developed for the proposed mathematical model, calculations were performed for a multilayer nanostructure with - AlN potential barriers and $\mathrm{GaN}$ - potential wells.

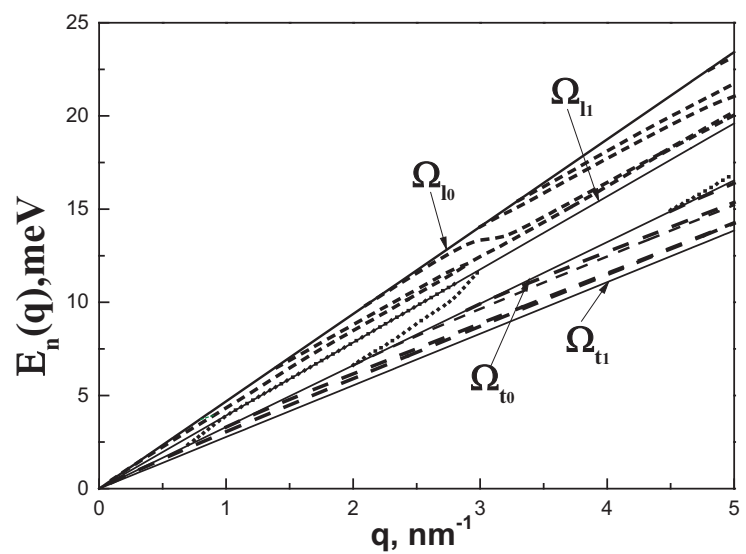

Fig. 2. Dependence of the acoustic phonons spectrum $E_{n}$ on the wave vector $q$

Direct calculations were performed for a three-well nanostructure with potential wells $3 \mathrm{~nm}$ thick and two potential barriers $2 \mathrm{~nm}$ thick. The physical parameters of the nanostructure materials are known from papers [7, 9].

In Fig. 2 the dependencies of the acoustic phonons spectrum $E_{n}$ as a function of the wave vector $q=1 / x_{3}^{N}$ are shown. It is seen from the figure that the dependency branches $E_{n}(q)$ grow with increasing of the wave vector $q$, forming three dependency groups with different dispersions, and the limiting values for each of the groups correspond to the dependences of the energies of longitudinal $\quad \Omega_{l_{0}}=\Omega_{l_{0}}(\mathrm{GaN}) ; \Omega_{l_{1}}=\Omega_{l_{1}}(\mathrm{AlN}) \quad$ and transverse $\quad \Omega_{t_{0}}=\Omega_{t_{0}}(\mathrm{GaN}) ; \Omega_{t_{1}}=\Omega_{t_{1}}(\mathrm{AlN}) \quad$ acoustic phonons in massive crystals.

In addition, as it can be seen from the figure, the dependences have a typical feature: the final energies of the acoustic phonons in each group begin at the energy of the longitudinal acoustic phonon, and end at the transverse phonon's value. A direct experimental verification of the acoustic phonon spectrum with the aim of comparing with the modeling results can be carried out by studying the Raman scattering of these phonons.

\section{CONCLUSION}

Proposed an analytical mathematical model on the basis of which a theory of the spectrum and normalized modes of acoustic phonons arising in a $\mathrm{AlN} / \mathrm{GaN}$ multilayer semiconductor nanosystem is developed. Based on the calculations performed using the proposed model for the geometric and physical parameters of a typical nanosystem, the spectral characteristics of the acoustic phonon modes and their dependence on the wave vector are obtained. The proposed model and theory are of a general nature and can be applied to the study and modeling of the spectrum and modes of acoustic phonons in nanosystems with different physical and geometrical parameters. Also, this mathematical model can be further used to study the interaction of various types of quasiparticles in semiconductor nanosystems.

\section{REFERENCES}

[1] Q.Y. Lu, S. Manna, D.H. Wu, S. Slivken, M. Razeghi, "Shortwave quantum cascade laser frequency comb for multi-heterodyne spectroscopy", Applied Physics Letters, vol. 112, no. 14, pp. 141104, 2018.

[2] F. Wang, X.G. Guo, J.C. Cao, "Transient energy relaxation in scattering-assisted terahertz quantum cascade lasers", Applied Physics Letters, vol. 110, no. 10, pp. 103505, 2017

[3] W. Rudno-Rudziński, D. Biegańska, J. Misiewicz, F. Lelarge, B. Rousseau, G. Sęk, "Carrier diffusion as a measure of carrier/exciton transfer rate in InAs/InGaAsP/InP hybrid quantum dot-quantum well structures emitting at telecom spectral range", Applied Physics Letters, vol. 112, no. 5, pp. 051103, 2017.

[4] L. Zhang, W. Zheng, A. Song, "Adaptive logical stochastic resonance in time-delayed synthetic genetic networks", Chaos, vol. 28, no. 4, pp. 043117, 2018

[5] Q. Liu, M. Li, K. Dai, K. Zhang, G. Xue, X. Tana, H. Yub, Y. Yu, "Extensible 3D architecture for superconducting quantum computing", Applied Physics Letters, vol. 110, no. 23, pp. 232602, 2017.

[6] R. Shugayev, P. Bermel, "Core-shell Mie resonant structures for quantum computing applications", Applied Physics Letters, vol. 109, no. 22, pp. 221102, 2016.

[7] C. Kumar N. Patel, R.Barron-Jimenez, I. Dunayevskiy, G. Tsvid, A. Lyakh, "Two wavelength operation of an acousto-optically tuned quantum cascade laser and direct measurements of quantum cascade laser level lifetimes", Applied Physics Letters, vol. 110, no. 3, pp. 031104, 2017.

[8] S. Singh, R. Kamoua, "Scattering assisted injection based injectorless mid infrared quantum cascade laser", Journal of Applied Physics, vol. 115, no. 21, pp. $213106,2014$.

[9] E.P.Pokatilov, D. L.Nika, A. A.Balandin, "Phonon spectrum and group velocities in AlN/GaN/AIN and related heterostructures", Superlattices and Microstructures, vol. 33, no. 3, pp. 155-171, 2003.

[10] M.V. Tkach, Ju.O. Seti, I.V. Boyko, O.M. Voitsekhivska, "Optimization of quantum cascade laser operation by geometric design of cascade active band in open and closed models", Condensed Matter Physics, vol. 16, no. 3, pp. 33701, 2013. 